\title{
The roles of cell cycle and BRCA1 in the DNA damage response. ${ }^{\dagger}$
}

\author{
Shihori Tanabe ${ }^{1, *}$, Sabina Quader ${ }^{2}$, Ryuichi Ono ${ }^{3}$, Horacio Cabral ${ }^{4}$, Kazuhiko Aoyagi ${ }^{5}$, Akihiko \\ Hirose ${ }^{1}$, Hiroshi Yokozaki ${ }^{6}$ and Hiroki Sasaki ${ }^{7}$
}

1 Division of Risk Assessment, Center for Biological Safety and Research, National Institute of Health Sciences, Kawasaki 210-9501, Japan; stanabe@nihs.go.jp (S.T.), hirose@nihs.go.jp (A.H.)

2 Innovation Center of NanoMedicine (iCONM), Kawasaki Institute of Industrial Promotion, Kawasaki 210-0821, Japan; sabina-q@kawasaki-net.ne.jp

3 Division of Cellular and Molecular Toxicology, Center for Biological Safety and Research, National Institute of Health Sciences, Kawasaki 210-9501, Japan; onoryu@nihs.go.jp

4 Department of Bioengineering, Graduate School of Engineering, University of Tokyo, Tokyo 113-0033, Japan; horacio@bmw.t.u-tokyo.ac.jp

5 Department of Clinical Genomics, National Cancer Center Research Institute, Tokyo 104-0045, Japan; kaaoyagi@ncc.go.jp

6 Department of Pathology, Kobe University of Graduate School of Medicine, Kobe 650-0017, Japan; hyoko@med.kobe-u.ac.jp

7 Department of Translational Oncology, National Cancer Center Research Institute, Tokyo 104-0045, Japan; hksasaki@ncc.go.jp

* Correspondence: stanabe@nihs.go.jp; Tel.: +81-44-270-6686

+ Presented at the 1st International Electronic Conference on Cancers: Exploiting Cancer Vulnerability by Targeting the DNA Damage Response, online, 01-14 February 2021.

Received: date; Accepted: date; Published: date

\begin{abstract}
In cancer stem cells (CSCs), epithelial-mesenchymal transition (EMT) networks play an important role to acquisition of the drug resistance and cancer malignant feature. To reveal the network pathways in EMT and CSCs, gene expression in diffuse- and intestinal-type gastric cancer (GC) have been analyzed. A canonical pathway on Cell Cycle: G1/S Checkpoint Regulation was activated in diffuse-type GC, and Cyclins and Cell Cycle Regulation was activated in intestinal-type GC. Canonical pathway related to Role of BRCA1 in DNA Damage Response was activated in intestinal-type GC, where BRCA1 which is related to $\mathrm{G}_{1} / \mathrm{S}$ phase transition was up-regulated. Cell cycle regulation may be altered in EMT condition.
\end{abstract}

Keywords: BRCA1; cancer stem cell; cell cycle; epithelial-mesenchymal transition; DNA damage response; molecular network

\section{Introduction}

In cancer stem cells (CSCs), epithelial-mesenchymal transition (EMT) networks play an important role to acquisition of the drug resistance and cancer malignant feature [1]. To reveal the network pathways in EMT and CSCs, gene expression in diffuse- and intestinal-type gastric cancer (GC) have been analyzed. Our previous findings identified several molecular networks and the related microRNAs (miRNAs) in intestinal- and diffuse-type GC [2-5]. Cell proliferation and regulation of the cell cycle are essential in cancer therapeutic targeting. In this article, we focus on the roles of cell cycle and BRCA1 in the DNA damage response in diffuse- and intestinal-type GC. Cell cycle regulation may play an important role in intestinal- and diffuse-type GC. The mechanism of cancer drug resistance would be highlighted by the involvement of cell cycle in EMT and CSCs.

\section{Materials and Methods}




\subsection{RefSeq data analysis}

The RefSeq data of diffuse-type and intestinal- GC are publicly available in The Cancer Genome Atlas (TCGA) of The cBioPortal for Cancer Genomics database [6-8] in NCI Genomic Data Commons (GDC) Data Portal [9]. From the data of stomach adenocarcinoma (TCGA, PanCancer Atlas), intestinal- and diffuse-type GC data, which are noted as chromosomal instability (CIN) and genomically stable (GS), respectively, in TCGA Research Network publication, were compared [8].

\subsection{Molecular genome network analysis}

Data of intestinal- and diffuse-type GC in TCGA cBioPortal Cancer Genomics were uploaded and analyzed through the use of Ingenuity Pathway Analysis (IPA) (QIAGEN Inc., Hilden, Germany) [10].

\subsection{Data Visualization}

The results of gene expression data of RefSeq and network analysis were visualized by Tableau software.

\section{Results and Discussion}

\subsection{Canonical pathways altered in diffuse- and intestinal-type GC}

Canonical pathways altered in diffuse- and intestinal-type GC are shown in Figure 1 and Table 1. The 2815 IDs which are significantly different in diffuse- and intestinal-type GC were analyzed in network analysis, which identified 69 canonical pathways related to the diffuse- and intestinal-type GC. Gene expression data of the diffuse- and intestinal-type GC revealed 36 canonical pathways with activation z-score as shown in Table 1 . These canonical pathways include Cell Cycle: $\mathrm{G}_{1} / \mathrm{S}$ Checkpoint Regulation, Cyclins and Cell Cycle Regulation, Role of BRCA1 in DNA Damage Response, and Cell Cycle: G2/M DNA Damage Checkpoint.

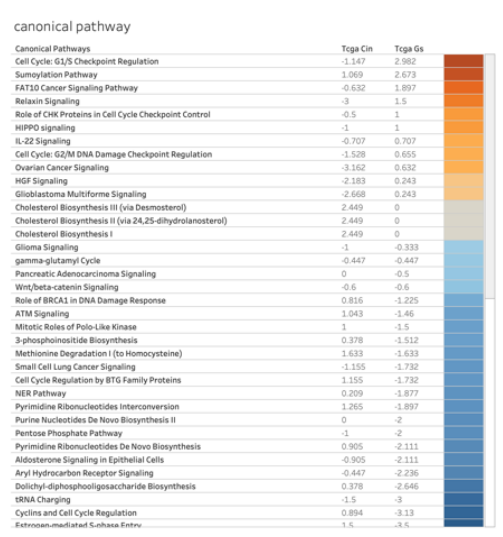

(a)

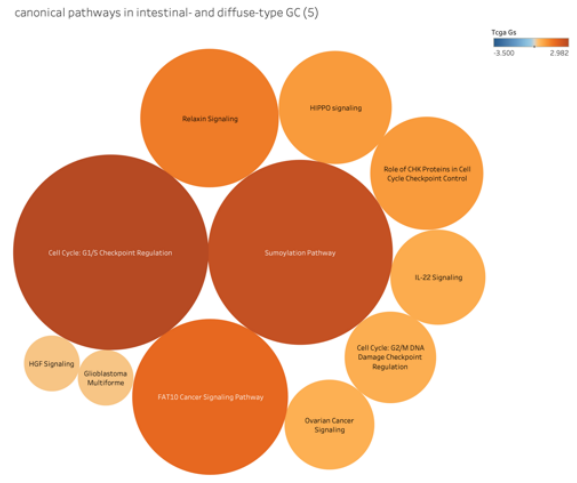

(c)

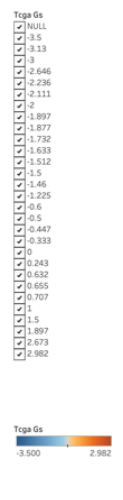

(b)

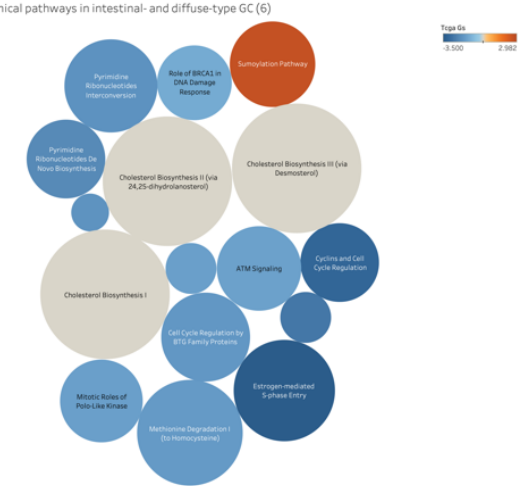

(d) 
Figure 1. Canonical pathways altered in diffuse- and intestinal-type GC are shown. (a) Canonical pathways sorted by the order of the activation z-score in diffuse-type GC are shown; (b) Canonical pathways sorted by the order of the p-value of the pathways are shown; (c) Size and color indicate the activation z-score in diffuse-type GC; (c) Size indicates the activation z-score in intestinal-type GC and color indicates the activation z-score in intestinal-type GC.

Table 1. Canonical pathways altered in diffuse- and intestinal-type GC. The pathways are sorted by the order of the activation $\mathrm{z}$-score.

\begin{tabular}{lll}
\hline Canonical Pathways & diffuse-type & intestinal-type \\
& GC & GC (activation \\
& (activation & z-score) \\
& z-score) & \\
\hline Cell Cycle: G1/S Checkpoint Regulation & 2.982 & -1.147 \\
Sumoylation Pathway & 2.673 & 1.069 \\
FAT10 Cancer Signaling Pathway & 1.897 & -0.632 \\
Relaxin Signaling & 1.5 & -3 \\
HIPPO signaling & 1 & -1 \\
Role of CHK Proteins in Cell Cycle Checkpoint & 1 & -0.5 \\
Control & & \\
IL-22 Signaling & 0.707 & -0.707 \\
Cell Cycle: G2/M DNA Damage Checkpoint & 0.655 & -1.528 \\
Regulation & & \\
Ovarian Cancer Signaling & 0.632 & -3.162 \\
Glioblastoma Multiforme Signaling & 0.243 & -2.668 \\
HGF Signaling & 0.243 & -2.183 \\
Cholesterol Biosynthesis II (via & 0 & 2.449 \\
24,25-dihydrolanosterol) & & \\
Cholesterol Biosynthesis III (via Desmosterol) & 0 & 2.449 \\
Cholesterol Biosynthesis I & 0 & 2.449 \\
Glioma Signaling & -0.333 & -1 \\
gamma-glutamyl Cycle & -0.447 & -0.447 \\
Pancreatic Adenocarcinoma Signaling & -0.5 & 0 \\
Wnt/beta-catenin Signaling & -0.6 & -0.6 \\
Role of BRCA1 in DNA Damage Response & -1.225 & 0.816 \\
ATM Signaling & -1.46 & 1.043 \\
Mitotic Roles of Polo-Like Kinase & -1.5 & 1 \\
3-phosphoinositide Biosynthesis & -1.512 & 0.378 \\
Methionine Degradation I (to Homocysteine) & -1.633 & 1.633 \\
Small Cell Lung Cancer Signaling & -1.732 & -1.155 \\
Cell Cycle Regulation by BTG Family Proteins & -1.732 & 1.155 \\
NER Pathway & -1.877 & 0.209 \\
Pyrimidine Ribonucleotides Interconversion & -1.897 & 1.265 \\
Pentose Phosphate Pathway & -2 & -1 \\
\hline & & \\
\hline
\end{tabular}




\begin{tabular}{lll}
\hline Purine Nucleotides De Novo Biosynthesis II & -2 & 0 \\
Aldosterone Signaling in Epithelial Cells & -2.111 & -0.905 \\
Pyrimidine Ribonucleotides De Novo & -2.111 & 0.905 \\
Biosynthesis & & \\
Aryl Hydrocarbon Receptor Signaling & -2.236 & -0.447 \\
Dolichyl-diphosphooligosaccharide & -2.646 & 0.378 \\
Biosynthesis & & \\
tRNA Charging & -3 & -1.5 \\
Cyclins and Cell Cycle Regulation & -3.13 & 0.894 \\
Estrogen-mediated S-phase Entry & -3.5 & 1.5 \\
\hline
\end{tabular}

\subsection{Cell Cycle: G1/S checkpoint Regulation pathway was activated in diffuse-type GC}

Molecule activity predictor in IPA predicted the activation of Cell Cycle: G1/S Checkpoint Regulation pathway in diffuse-type GC (Figure 2). In Cell Cycle: G1/S Checkpoint Regulation pathway, DNA damage induces p53, which is expected to be activated in diffuse-type GC. Analysis of direct relationships of miRNAs and targeted molecules in Cell Cycle: G1/S Checkpoint Regulation pathway revealed the relationships between miRNAs and the targeted molecules (Figure 2c, Table 2).

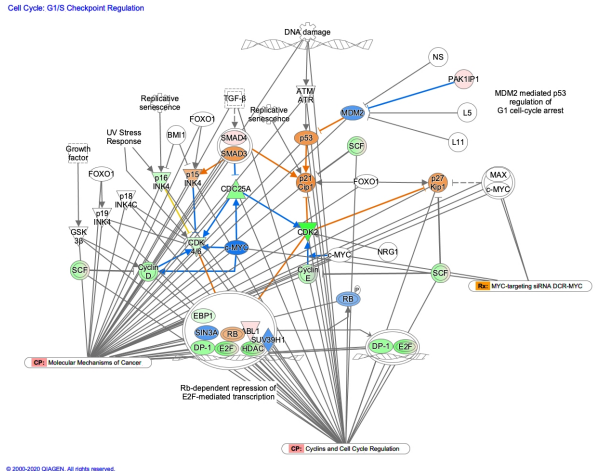

(a)

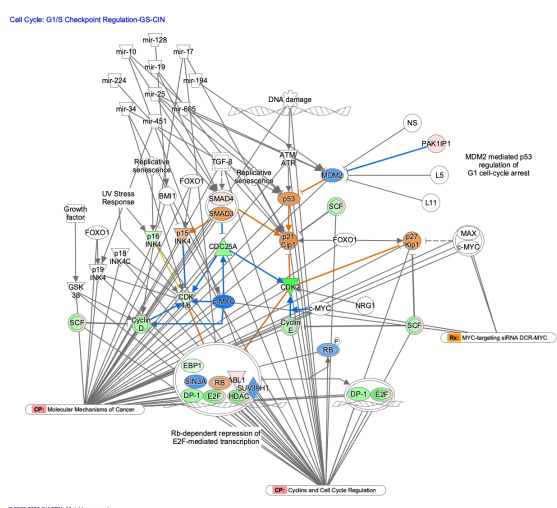

(c)

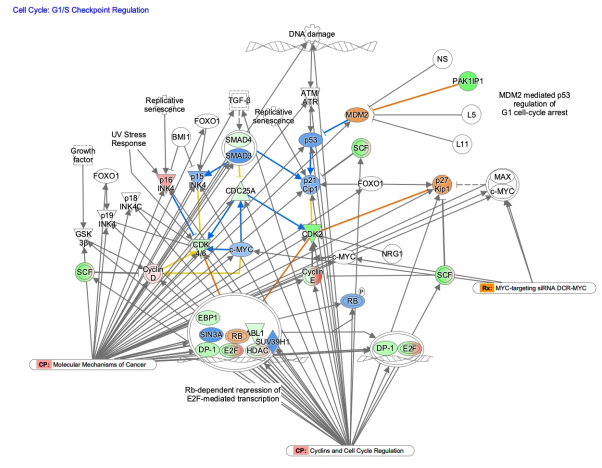

(b)

Figure 2. Cell Cycle: G1/S Checkpoint Regulation pathway was activated in diffuse-type GC. (a) Gene expression and pathway activity prediction in diffuse-type GC are shown; (b) Gene expression and pathway activity prediction in intestinal-type GC are shown; (c) Direct relationships of miRNAs and targeted molecules in the pathway are shown. The genes of which expression was altered in diffuse- 
and intestinal-type GC are shown in pink (up-regulated) or green (down-regulated). Predicted activation or inhibition is shown in orange or blue, respectively.

Table 2. Direct relationships of miRNAs and targeted molecules in Cell Cycle: G1/S Checkpoint Regulation pathway.

\begin{tabular}{ll}
\hline From Molecule(s) & To Molecule(s) \\
\hline mir-10 & SMAD4 \\
mir-10 & SUV39H1 \\
mir-10 & p53 \\
mir-128 & BMI1 \\
mir-17 & CyclinD \\
mir-17 & RB \\
mir-17 & p21Cip1 \\
mir-19 & SMAD4 \\
mir-19 & p21Cip1 \\
mir-194 & MDM2 \\
mir-224 & SMAD4 \\
mir-25 & MDM2 \\
mir-25 & p21Cip1 \\
mir-25 & p53 \\
mir-34 & CDK4/6 \\
mir-34 & c-MYC \\
mir-34 & p53 \\
mir-451 & p19INK4 \\
mir-605 & MDM2 \\
\hline
\end{tabular}

\subsection{Cyclins and Cell Cycle Regulation pathway was activated in intestinal-type GC}

Molecule activity predictor in IPA predicted the activation of Cyclins and Cell Cycle Regulation pathway in intestinal-type GC (Figure 3). Analysis of direct relationships of miRNAs and targeted molecules in Cyclins and Cell Cycle Regulation pathway revealed the relationships between miRNAs and the targeted molecules (Figure 3c, Table 3). 


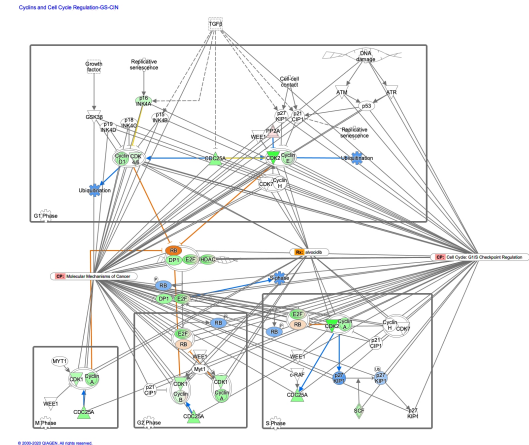

(a)

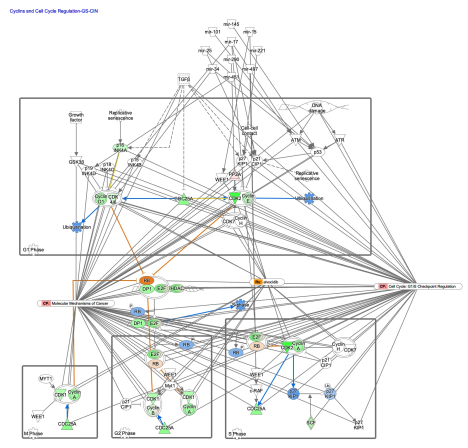

(c)

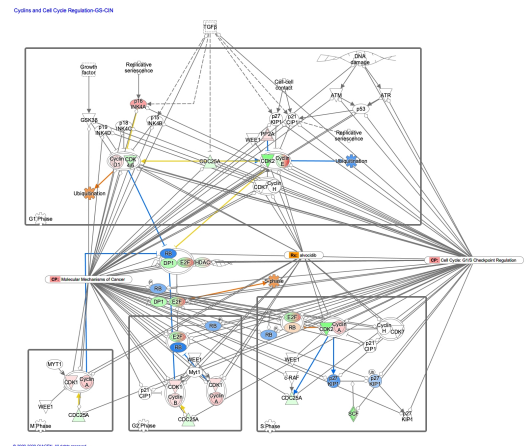

(b)

Figure 3. Cyclins and Cell Cycle Regulation pathway was activated in intestinal-type GC. (a) Gene expression and pathway activity prediction in diffuse-type GC are shown; (b) Gene expression and pathway activity prediction in intestinal-type GC are shown; (c) Direct relationships of miRNAs and targeted molecules in the pathway are shown. The genes of which expression was altered in diffuseand intestinal-type GC are shown in pink (up-regulated) or green (down-regulated). Predicted activation or inhibition is shown in orange or blue, respectively.

Table 3. Direct relationships of miRNAs and targeted molecules in Cyclins and Cell Cycle Regulation pathway.

\begin{tabular}{ll}
\hline From Molecule(s) & To Molecule(s) \\
\hline mir-101 & ATM \\
mir-145 & p53 \\
mir-15 & WEE1 \\
mir-15 & c-RAF \\
mir-17 & ATM \\
mir-17 & CyclinD1 \\
mir-17 & RB \\
mir-17 & p21CIP1 \\
mir-221 & p27KIP1 \\
mir-25 & p21CIP1 \\
mir-25 & p53 \\
mir-290 & CDK2 \\
mir-34 & CDK4/6 \\
mir-34 & p53 \\
\hline
\end{tabular}




\begin{tabular}{ll}
\hline mir-451 & p19INK4D \\
mir-497 & c-RAF \\
\hline
\end{tabular}

\subsection{Role of BRCA1 in DNA Damage Response pathway was activated in intestinal-type GC}

Molecule activity predictor in IPA predicted the activation of Role of BRCA1 in DNA Damage Response pathway in intestinal-type GC (Figure 4). Role of BRCA1 in DNA Damage Response pathway was identified as the most significant canonical pathway with $p$ value of $6.6 \times 10^{-12}$. Gene expression of BRCA1 which is associated with $\mathrm{G}_{1} / \mathrm{S}$ transition has increased in intestinal-type GC. BRCA1 codes a 190kD nuclear phosphorylation protein that maintains genomic stability and functions as a tumor suppressor. It is interesting that p53 and c21CIP1 are activate in intestinal-type GC in the Role of BRCA1 in DNA Damage Response pathway. BRCA1 may be involved in the activation of p53. Analysis of direct relationships of miRNAs and targeted molecules in Role of BRCA1 in DNA Damage Response pathway revealed the relationships between miRNAs and the targeted molecules (Figure 4c, Table 4). Ten miRNAs which have direct relationships between BRCA1 in Role of BRCA1 in DNA Damage Response pathway included miR-125a-3p (miRNAs w/seed CAGGUGA), miR-146a-5p (and other miRNAs w/seed GAGAACU), miR-224-5p (miRNAs w/seed AAGUCAC), miR-3615 (miRNAs w/seed CUCUCGG), miR-4639-3p (and other miRNAs w/seed CACUCUC), miR-5586-3p (miRNAs w/seed AGAGUGA), miR-6516-5p (miRNAs w/seed UUGCAGU), miR-6814-5p (miRNAs w/seed CCCAAGG), miR-6875-3p (miRNAs w/seed UUCUUCC), miR-99a-3p (and other miRNAs w/seed AAGCUCG) (Figure 4d, Table 5).

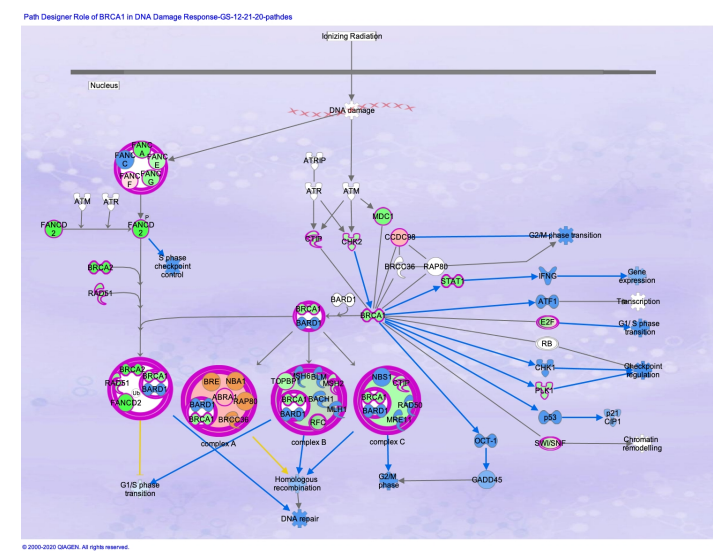

(a)

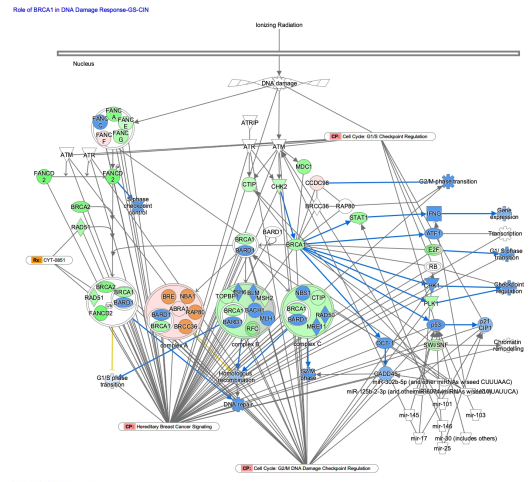

(c)

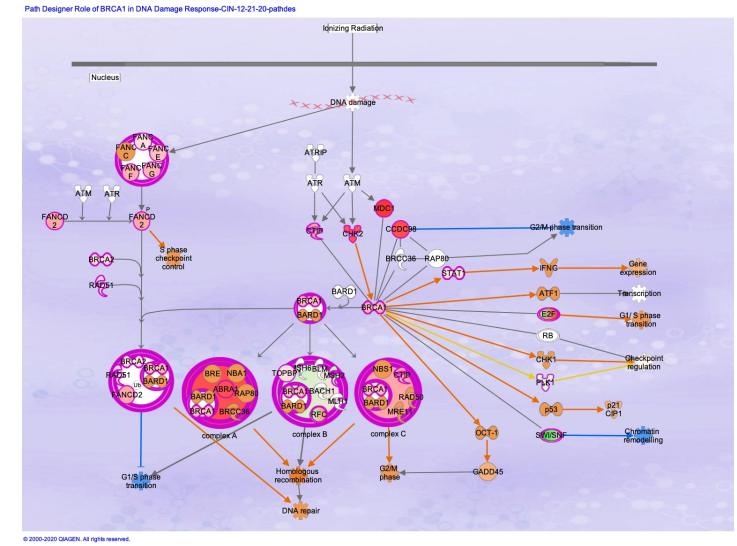

(b)

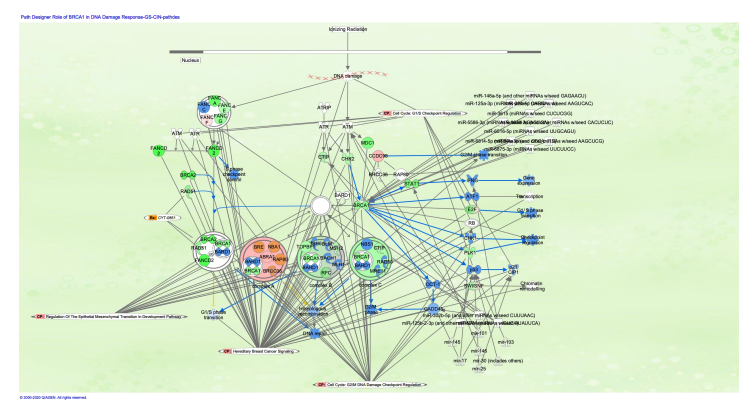

(d)

Figure 4. Role of BRCA1 in DNA Damage Response pathway was activated in intestinal-type GC. (a) Gene expression and pathway activity prediction in diffuse-type GC are shown; (b) Gene expression and pathway activity prediction in intestinal-type GC are shown; (c) Direct relationships of miRNAs and targeted molecules in the pathway are shown. (d) Direct relationships of miRNAs and BRCA1 in 
the pathway in diffuse-type GC are shown. The genes of which expression was altered in diffuseand intestinal-type GC are shown in pink (up-regulated) or green (down-regulated). Predicted activation or inhibition is shown in orange or blue, respectively.

Table 4. Direct relationships of miRNAs and targeted molecules in Role of BRCA1 in DNA Damage Response pathway.

\begin{tabular}{ll}
\hline From Molecule(s) & To Molecule(s) \\
\hline miR-125b-2-3p (and other miRNAs w/seed CAAGUCA) & p53 \\
miR-302b-5p (and other miRNAs w/seed CUUUAAC) & BARD1 \\
miR-302b-5p (and other miRNAs w/seed CUUUAAC) & CTIP \\
miR-302b-5p (and other miRNAs w/seed CUUUAAC) & GADD45 \\
miR-6074 (miRNAs w/seed AUAUUCA) & FANCF \\
miR-6074 (miRNAs w/seed AUAUUCA) & IFNG \\
miR-6074 (miRNAs w/seed AUAUUCA) & NBS1 \\
mir-101 & ATM \\
mir-103 & p53 \\
mir-145 & p53 \\
mir-146 & STAT1 \\
mir-17 & ATM \\
mir-17 & RB \\
mir-17 & p21CIP1 \\
mir-25 & p21CIP1 \\
mir-25 & p53 \\
mir-30 (includes others) & p53 \\
\hline
\end{tabular}

Table 5. Ten miRNAs which have direct relationships between BRCA1 in Role of BRCA1 in DNA Damage Response pathway.

\begin{tabular}{l}
\hline \multicolumn{1}{c}{ Ten miRNAs which have direct relationships between } \\
BRCA1 \\
\hline miR-125a-3p (miRNAs w/seed CAGGUGA) \\
miR-146a-5p (and other miRNAs w/seed GAGAACU) \\
miR-224-5p (miRNAs w/seed AAGUCAC) \\
miR-3615 (miRNAs w/seed CUCUCGG) \\
miR-4639-3p (and other miRNAs w/seed CACUCUC) \\
miR-5586-3p (miRNAs w/seed AGAGUGA) \\
miR-6516-5p (miRNAs w/seed UUGCAGU) \\
miR-6814-5p (miRNAs w/seed CCCAAGG) \\
miR-6875-3p (miRNAs w/seed UUCUUCC) \\
miR-99a-3p (and other miRNAs w/seed AAGCUCG) \\
\hline
\end{tabular}


Cell Cycle: G2/M DNA Damage Checkpoint Regulation pathway was identified as related canonical pathway in diffuse- and intestinal- type GC (Figure 5). Analysis of direct relationships of miRNAs and targeted molecules in Cell Cycle: G2/M DNA Damage Checkpoint Regulation pathway revealed the relationships between miRNAs and the targeted molecules (Figure 5c, Table 6).

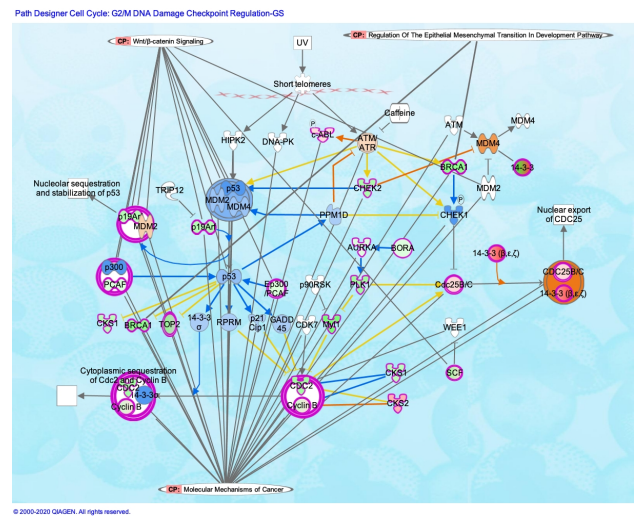

(a)

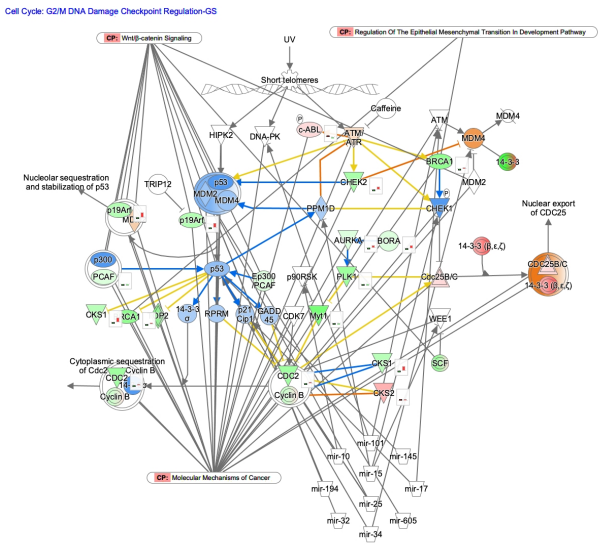

(c)

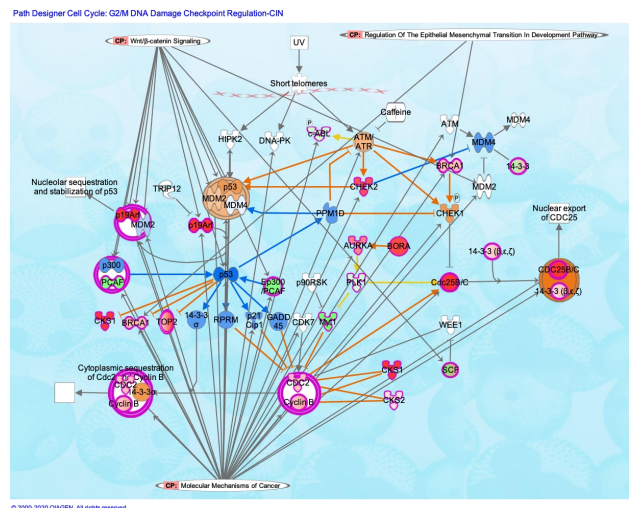

(b)

Figure 5. Cell Cycle: G2/M DNA Damage Checkpoint Regulation pathway in diffuse- and intestinal-type GC. (a) Gene expression and pathway activity prediction in diffuse-type GC are shown; (b) Gene expression and pathway activity prediction in intestinal-type GC are shown; (c) Direct relationships of miRNAs and targeted molecules in the pathway are shown. The genes of which expression was altered in diffuse- and intestinal-type GC are shown in pink (up-regulated) or green (down-regulated). Predicted activation or inhibition is shown in orange or blue, respectively.

Table 6. Direct relationships of miRNAs and targeted molecules in Cell Cycle: G2/M DNA Damage Checkpoint Regulation pathway.

\begin{tabular}{ll}
\hline From Molecule(s) & To Molecule(s) \\
\hline mir-10 & p53 \\
mir-10 & p90RSK \\
mir-101 & ATM \\
mir-101 & DNA-PK \\
mir-145 & p53 \\
\hline
\end{tabular}




\begin{tabular}{ll}
\hline mir-15 & CHEK1 \\
mir-15 & PPM1D \\
mir-15 & WEE1 \\
mir-17 & ATM \\
mir-17 & p21Cip1 \\
mir-194 & MDM2 \\
mir-25 & MDM2 \\
mir-25 & p21Cip1 \\
mir-25 & p53 \\
mir-32 & MDM2 \\
mir-34 & MDM4 \\
mir-34 & p53 \\
mir-605 & MDM2 \\
\hline
\end{tabular}

\section{Conclusion}

The several canonical pathways have been found to be altered in diffuse- and intestinal-type GC. Canonical pathway on Cell Cycle: G1/S Checkpoint Regulation was activated in diffuse-type GC, and Cyclins and Cell Cycle Regulation was activated in intestinal-type GC. Canonical pathway related to Role of BRCA1 in DNA Damage Response was activated in intestinal-type GC, where BRCA1 which is related to $G_{1} / S$ phase transition was up-regulated. Cell cycle regulation may be altered in EMT condition in diffuse-type GC.

Author Contributions: Conceptualization, S.T. and H.S.; methodology, S.T.; software, S.T.; formal analysis, S.T.; investigation, S.T.; data curation, S.T., K.A. and H.S.; writing-original draft preparation, S.T.; writing-review and editing, S.T., S.Q. and H.C.; visualization, S.T.; supervision, S.T. and A.H.; project administration, S.T., K.A., H.Y. and H.S.; funding acquisition, S.T., S.Q., R.O. and A.H. All authors have read and agreed to the published version of the manuscript.

Funding: This research was funded by Japan Agency for Medical Research and Development (AMED), grant number JP20ak0101093 (ST, RO and AH) and JP20mk0101163 (RO), and Strategic International Collaborative Research Program, grant number JP20jm0210059 (ST and SQ), Ministry of Health, Labour, and Welfare (MHLW), grant number H30-KAGAKU-IPPAN-002 (ST and RO), and JSPS KAKENHI grant number 18K19315 (RO).

Acknowledgments: The authors would like to thank all colleagues for their support.

Conflicts of Interest: The authors declare no conflict of interest.

\section{References}

1. Shibue, T.; Weinberg, R.A. EMT, CSCs, and drug resistance: the mechanistic link and clinical implications. Nat Rev Clin Oncol 2017, 14, 611-629, doi:10.1038/nrclinonc.2017.44.

2. Tanabe, S.; Quader, S.; Ono, R.; Cabral, H.; Aoyagi, K.; Hirose, A.; Yokozaki, H.; Sasaki, H. Molecular Network Profiling in Intestinal- and Diffuse-Type Gastric Cancer. Cancers 2020, 12, doi:10.3390/cancers12123833.

3. Tanabe, S.; Aoyagi, K.; Yokozaki, H.; Sasaki, H. Gene expression signatures for identifying diffuse-type gastric cancer associated with epithelial-mesenchymal transition. Int J Oncol 2014, 44, 1955-1970, doi:10.3892/ijo.2014.2387.

4. Tanabe, S.; Kawabata, T.; Aoyagi, K.; Yokozaki, H.; Sasaki, H. Gene expression and pathway analysis of CTNNB1 in cancer and stem cells. World J Stem Cells 2016, 8, 384-395, doi:10.4252/wjsc.v8.i11.384. 
5. Tanabe, S.; Quader, S.; Cabral, H.; Ono, R. Interplay of EMT and CSC in Cancer and the Potential Therapeutic Strategies. Front Pharmacol 2020, 11, 904, doi:10.3389/fphar.2020.00904.

6. Cerami, E.; Gao, J.; Dogrusoz, U.; Gross, B.E.; Sumer, S.O.; Aksoy, B.A.; Jacobsen, A.; Byrne, C.J.; Heuer, M.L.; Larsson, E., et al. The cBio Cancer Genomics Portal: An Open Platform for Exploring Multidimensional Cancer Genomics Data. Cancer Discovery 2012, 2, 401, doi:10.1158/2159-8290.CD-12-0095.

7. Gao, J.; Aksoy, B.A.; Dogrusoz, U.; Dresdner, G.; Gross, B.; Sumer, S.O.; Sun, Y.; Jacobsen, A.; Sinha, R.; Larsson, E., et al. Integrative analysis of complex cancer genomics and clinical profiles using the cBioPortal. Sci Signal 2013, 6, pl1, doi:10.1126/scisignal.2004088.

8. Bass, A.J.; Thorsson, V.; Shmulevich, I.; Reynolds, S.M.; Miller, M.; Bernard, B.; Hinoue, T.; Laird, P.W.; Curtis, C.; Shen, H., et al. Comprehensive molecular characterization of gastric adenocarcinoma. Nature 2014, 513, 202-209, doi:10.1038/nature13480.

9. Grossman, R.L.; Heath, A.P.; Ferretti, V.; Varmus, H.E.; Lowy, D.R.; Kibbe, W.A.; Staudt, L.M. Toward a Shared Vision for Cancer Genomic Data. $N$ Engl J Med 2016, 375, 1109-1112, doi:10.1056/NEJMp1607591.

10. Krämer, A.; Green, J.; Pollard, J., Jr.; Tugendreich, S. Causal analysis approaches in Ingenuity Pathway Analysis. Bioinformatics 2014, 30, 523-530, doi:10.1093/bioinformatics/btt703.

(C) 2020 by the authors. Submitted for possible open access publication under the terms and conditions of the Creative Commons Attribution (CC BY) license (http://creativecommons.org/licenses/by/4.0/). 